\title{
Intervención nutricional prequirúrgica en pacientes de cirugía colorrectal manejados con el protocolo ERAS
}

\author{
Pre-surgical nutritional intervention in colorectal surgery \\ patients managed with the ERAS protocol \\ Intervenção nutricional pré-cirúrgica em pacientes submetidos a \\ cirurgia colorretal tratados com o protocolo ERAS
}

\author{
Ángela Navas Camacho ${ }^{1 *}$, Iveth Pérez Díaz', Gustavo Alfonso Díaz Muñoz ${ }^{2}$
}

Recibido: 1 de septiembre de 2019. Aceptado para publicación: 20 de enero de 2020 https://doi.org/10.35454/rncm.v3n1.083

\begin{abstract}
Resumen
Antecedentes: la intervención nutricional perioperatoria en el marco del protocolo ERAS se ha posicionado como estrategia costo efectiva para el manejo de pacientes de cirugía mayor.

Objetivo: describir la intervención nutricional prequirúrgica según el protocolo ERAS en pacientes adultos sometidos a cirugía colorrectal programada en la Clínica Reina Sofía en Bogotá entre marzo de 2016 y diciembre de 2018.
\end{abstract}

Métodos: estudio observacional, descriptivo, transversal y de prevalencia de periodo. Se evaluaron los pacientes de cirugía colorrectal manejados con el protocolo ERAS en la Clínica Reina Sofía entre marzo de 2016 y diciembre de 2018.

Resultados: el protocolo ERAS se aplicó a 181 pacientes, la edad media 63,9 \pm 14,8 años, $42 \%$ eran hombres. En la consulta prequirúrgica se encontró que 9,9\% eran diabéticos, tenían un promedio de IMC $25,2 \pm 4,0 \mathrm{~kg} / \mathrm{m}^{2}$ y el $13,8 \%$ y $8,8 \%$ presentó riesgo de desnutrición y desnutrición respectivamente. Acerca de las intervenciones nutricionales prequirúrgicas que recibieron los pacientes, 76,8 \% recibieron fórmulas inmunomoduladoras, $13,8 \%$ suplementos nutricionales estándar y 6,6 \% alimentación habitual. De los pacientes operados 6,6\% presentó íleo paralítico y $6,1 \%$ fuga anastomótica.

\section{Summary}

Background: The perioperative nutritional intervention under the ERAS protocol has been positioned as a cost-effective strategy for the management of patients undergoing major surgery.

Objective: To describe the preoperative nutritional intervention according to the ERAS protocol in adult patients undergoing scheduled colorectal surgery at the Reina Sofía Clinic in Bogotá between March 2016 and December 2018.

Methods: Observational, descriptive, cross-sectional and period prevalence study. Colorectal surgery patients managed with the ERAS protocol were evaluated at the Reina Sofía Clinic between March 2016 and December 2018.

Results: The ERAS protocol was applied to 181 patients. Mean age was $63,9 \pm 14.8$ years and $42 \%$ were men. Preoperative consultation showed that 9,9\% patients were diabetic, had an average BMI of 25,2 $\pm 4,0 \mathrm{~kg} / \mathrm{m}^{2}$, and had either a risk of malnutrition or overt malnutrition (13,8\% and $8,8 \%$, respectively). Regarding preoperative nutritional interventions administered $76,8 \%$ patients received immunomodulatory formulas, $13,8 \%$ standard nutritional supplements, and $6,6 \%$ regular feeding. During the postoperative period, $6,6 \% \mathrm{pa}$ tients presented paralytic ileus and 6,1\% anastomotic leak.

\section{Resumo}

Antecedentes: a intervenção nutricional perioperatória sob o protocolo ERAS foi posicionada como uma estratégia de custo eficaz, para o manejo de pacientes cirúrgicos de grande porte.

Objetivo: descrever a intervenção nutricional pré-cirúrgica de acordo com o protocolo ERAS em pacientes adultos submetidos a cirurgia colorretal programada na Clínica Reina Sofía em Bogotá entre março de 2016 e dezembro de 2018.

Métodos: estudo observacional, descritivo, transversal e de prevalência de período. Os pacientes submetidos à cirurgia colorretal tratados com o protocoIo ERAS foram avaliados na Clínica Reina Sofía entre março de 2016 e dezembro de 2018.

Resultados: o protocolo ERAS foi aplicado a 181 pacientes, com idade média de $63,9 \pm 14,8$ anos, $42 \%$ eram homens. Na consulta pré-cirúrgica, verificou-se que 9,9\% eram diabéticos, com IMC médio de $25,2 \pm 4,0 \mathrm{~kg} / \mathrm{m}^{2}$ e $97,8 \%$ com cirurgia programada; além disso, $13,8 \%$ e $8,8 \%$ apresentaram risco de desnutrição e desnutrição, respetivamente. Sobre as intervenções nutricionais pré-cirúrgicas que os pacientes receberam, 76,8 \% receberam fórmulas imunomoduladoras, $13,8 \%$ suplementos nutricionais padrão e $6,6 \%$ alimentação usual. Dos pacientes operados, 
Conclusiones: La implementación del protocolo ERAS en la Clínica Reina Sofía permitió que todos los pacientes adultos sometidos a cirugía colorrectal tuvieran una intervención nutricional estructurada. Se sugieren estudios que permitan valorar el impacto de las intervenciones de este protocolo sobre los desenlaces de los pacientes.

Palabras clave: periodo preoperatorio, cuidados perioperatorios, estado nutricional, recuperación mejorada después de la cirugía, soporte nutricional.
Conclusions: Implementation of the ERAS protocol at the Reina Sofía Clinic allowed adult patients undergoing colorectal surgery to receive a structured nutritional intervention. The undertaking of studies assessing the impact of interventions from ERAS protocol on patient outcomes is suggested.

Keywords: Preoperative period; Perioperative care; Nutritional status; Improved recovery after surgery; Nutritional support.
6,6 \% apresentaram íleo paralítico e 6,1 \% de vazamento anastomótico.

Conclusões: o protocolo ERAS foi aplicado a todos os pacientes: triagem nutricional, diagnóstico e intervenção nutricional no pré-operatório, o que foi realizado na maioria das vezes com o uso de fórmulas imunomodulatórias orais.

Palavras-chave: período pré-operatório, cuidados perioperatórios, estado nutricional, recuperação melhorada após a cirurgia, suporte nutricional.
Clínica Reina Sofía, Bogotá, D.C., Colombia.

*Correspondencia: Ángela Navas Camacho angelamarianavas@gmail.com

\section{INTRODUCCIÓN}

Desde el momento en que la desnutrición hospitalaria fue puesta en la mira del ámbito científico en 1974 por la investigación del Dr. Charles Butterworth y su artículo "El esqueleto en el armario del hospital"(1) y reconocida como factor de riesgo para complicaciones, aumento en la estancia hospitalaria, reingresos, fracasos terapéuticos e incremento en los costos de salud ${ }^{(2-4)}$, se fue despertando la conciencia de su impacto en diferentes patologías ${ }^{(5-8)}$.

El paciente quirúrgico no fue la excepción, y en efecto, los pacientes sometidos a cirugías mayores tienen una alta incidencia de desnutrición, la cual se considera como un factor de riesgo independiente que incrementa la morbimortalidad perioperatoria ${ }^{(9,10)}$, pero que responde positivamente a la intervención nutricional prequirúrgica, mejorando el pronóstico y disminuyendo el riesgo de complicaciones ${ }^{(11,12)}$. En la actualidad, la incidencia de malnutrición en el paciente quirúrgico se observa en la mayoría de los estudios entre $40 \%$ y $50 \%{ }^{(13)}$, siendo más alta en pacientes adultos mayores, enfermedad oncológica y patología gastrointestinal alta.

El protocolo ERAS $^{\circledast}$ (Enhanced Recovery After Surgery) es un modelo de cuidado perioperatorio multimodal, multidisciplinario y basado en la evidencia, que busca reorganizar la atención del paciente quirúrgico con
Instituto de Investigación en Nutrición, Genética y Metabolismo. Facultad de Medicina. Universidad El Bosque. Bogotá, D.C., Colombia. el fin de mejorar desenlaces mediante la adherencia a estrategias pre, intra y postoperatorias ${ }^{(14)}$. Como parte de las estrategias ERAS del periodo preoperatorio, las intervenciones nutricionales hacen parte del proceso de prehabilitación que busca mejorar las reservas fisiológicas y enfrentar en mejor condición el procedimiento quirúrgico $^{(13)}$. Este proceso nutricional incluye detección del riesgo, formulación de diagnóstico nutricional y planteamiento de manejo según la condición del paciente.

Parte del éxito en los resultados postoperatorios dependen de la modificación de las condiciones prequirúrgicas para impactar en forma positiva los desenlaces en cirugía. Las intervenciones nutricionales durante el periodo prequirúrgico son reconocidas como estrategias que modifican con certeza el efecto deletéreo de la desnutrición como factor de riesgo independiente para malos desenlaces ${ }^{(12)}$.

Por todo lo anterior, la Clínica Reina Sofía implementó el protocolo ERAS a partir de marzo de 2016 (Figura 1), con el fin de mejorar los desenlaces quirúrgicos, establecer mejores prácticas y contar con un sistema de auditoría a los procesos. En consecuencia, el objetivo de esta investigación fue describir las intervenciones nutricionales prequirúrgicas del proceso de atención nutricional del protocolo ERAS, en pacientes adultos sometidos a cirugía colorrectal programada en la Clínica Reina Sofía en la ciudad de Bogotá, D.C., Colombia. 


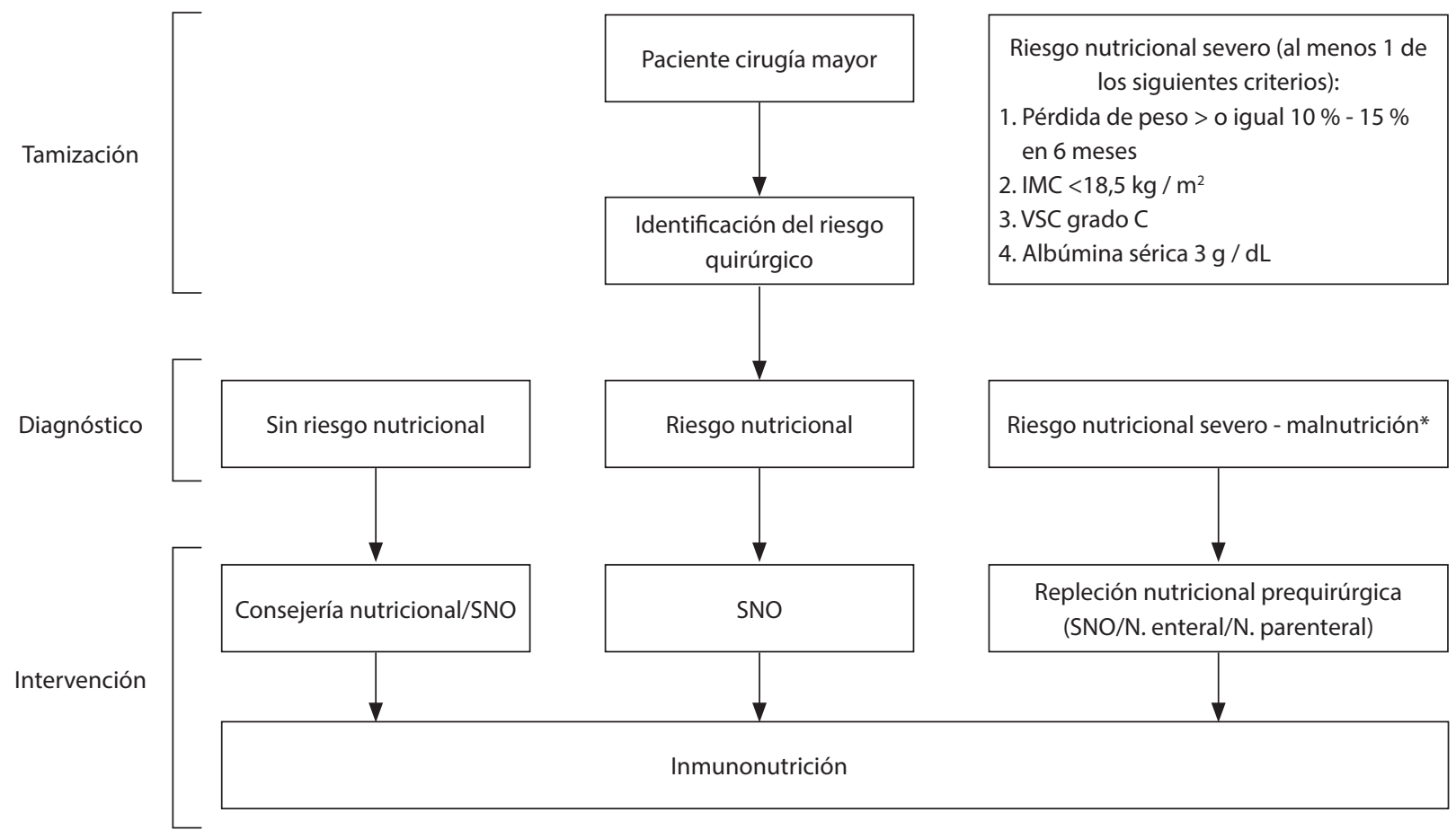

Figura 1. Protocolo ERAS para pacientes quirúrgicos en la Clínica Reina Sofía, Bogotá, D.C., Colombia. SNO: Suplemento Nutricional Oral; VSG: Valoración Global Subjetiva; IMC: Índice de Masa Corporal.

\section{METODOLOGÍA}

\section{Diseño del estudio}

Se diseñó un estudio observacional, descriptivo, transversal y de prevalencia de periodo (marzo 2016 a diciembre 2018). El estudio fue autorizado por la comisión de Investigación y el Comité de Ética de la institución.

\section{Población}

Se incluyeron en el estudio todos los pacientes adultos que fueron atendidos en la Clínica Reina Sofía para cirugía colorrectal programada manejados dentro del programa ERAS. Se excluyeron aquellos pacientes intervenidos de urgencia, pacientes pediátricos y pacientes de cirugía programada que no fueron incluidos en el programa ERAS de la Clínica Reina Sofía.

\section{Recolección de información}

La información se obtuvo de dos fuentes:
1. El sistema EIAS (ERAS Interactive Audit System) que fue desarrollado por $\mathrm{ENCARE}^{\oplus}$ para la sociedad ERAS ${ }^{\oplus}$ como una interfase interactiva y fácil de usar para la recolección de más de 100 variables del manejo del periodo perioperatorio de los pacientes de cirugía en donde se obtiene retroalimentación inmediata y una manera de vigilar objetivamente el desempeño, las mejoras y la calidad de la atención en cada una de las fases del periodo perioperatorio.

2. La historia clínica de los pacientes desarrollada por el grupo de consulta prequirúrgica conformado por profesionales en enfermería, nutrición y terapia ${ }^{(14)}$.

Se obtuvieron datos de los pacientes relacionados en la historia clínica de ingreso, los datos de la herramienta de detección de riesgo nutricional (MST y NRS) y los de la valoración nutricional prequirúrgica. También se tomaron datos relacionados con los desenlaces y complicaciones presentadas en el periodo postoperatorio principalmente fuga anastomótica. 
En el caso del IMC, la clasificación se hizo conforme a los parámetros de la OMS en los adultos $<60$ años (bajo peso: $<18,5 \mathrm{~kg} / \mathrm{m}^{2}$; normal: 18,5 a $24,9 \mathrm{~kg} / \mathrm{m}^{2}$; sobrepeso: $\geq 25 \mathrm{~kg} / \mathrm{m}^{2}$ ); para los adultos de 60 y más años se aplicaron los puntos de corte de la OPS (bajo peso: $<23 \mathrm{~kg} / \mathrm{m}^{2}$; normal: 23 a $27,9 \mathrm{~kg} / \mathrm{m}^{2}$; sobrepeso $\left.\geq 28 \mathrm{~kg} / \mathrm{m}^{2}\right)^{(14)}$.

\section{Protocolo ERAS}

El protocolo ERAS se implementó en marzo 2016 en la Clínica Reina Sofía de Bogotá, institución de tercer nivel, cumpliendo los lineamientos del programa ERAS Society ${ }^{\otimes}$. Estos incluyen la conformación de un "grupo implementador", la creación de un protocolo propio de la institución, la socialización en todos los grupos asistenciales y administrativos que participan en el manejo de los pacientes y la inclusión en el sistema interactivo de auditoría (EIAS) de 50 pacientes consecutivos, previos a la implementación del protocolo con el objetivo de tener una línea de base de comparación del antes y el después de la implementación. Con EIAS se hace el monitoreo de la adherencia a cada una de las estrategias.

El proceso de cuidado nutricional en el marco del protocolo ERAS inicia cuando el cirujano tratante valora el paciente y determina el requerimiento de una intervención quirúrgica específica. Posteriormente, es contactado por la jefe de enfermería coordinadora del Grupo ERAS para definir la fecha de valoración por tres profesionales de la salud: el profesional en enfermería quien realiza la consejería prequirúrgica, el profesional de nutrición clínica encargado de realizar el proceso de valoración e intervención nutricional y los profesionales de terapia física y respiratoria que realizan la evaluación de la condición física del paciente y diseñan la intervención prequirúrgica correspondiente. La consulta se realiza, según los lineamientos de ERAS, por lo menos diez a quince días antes del procedimiento para establecer una intervención nutricional en un tiempo adecuado. La información es consignada en EIAS.

La valoración del estado nutricional es realizada por la nutricionista. En primera instancia se realizó la determinación del riesgo nutricional aplicando una herramienta de tamizaje NRS 2002 o MST. En el inicio del programa ERAS no se tenía clara la preferencia por una herramienta de tamizaje nutricional en particular, se empezaron a utilizar las dos enunciadas previamente, pero se optó por la utilización de MST por ser más expedita. Posteriormente se realizó la anamnesis y valoración nutricional (antropométrica y funcional con dinamometría). El EIAS establece tres categorías para clasificar los pacientes desde el punto de vista nutricional: paciente bien nutrido, paciente con riesgo de desnutrición (determinado por puntaje mayor o igual a 2 en la escala MST y mayor o igual a 3 en NRS 2002) y paciente desnutrido según criterios de ESPEN: pérdida de peso $>10 \%-15 \%$ en los últimos seis meses, IMC $<18,5 \mathrm{~kg} / \mathrm{m}^{2}$, Valoración Global Subjetiva grado C o NRS $2002>5$ y albúmina sérica prequirúrgica $<3 \mathrm{~g} / \mathrm{dL}$ (sin evidencia de disfunción renal o hepática) ${ }^{(12)}$.

Una vez clasificado el estado nutricional en el que se encontraba el paciente, se definió el tipo de intervención nutricional: a. los pacientes sin riesgo recibieron consejería nutricional y fórmula inmunomoduladora 5 a 7 días antes de la cirugía; b. los pacientes con riesgo nutricional recibieron consejería y suplementación nutricional con fórmulas de alta densidad calórica con el fin de mejorar los aportes calóricos y proteicos con cambio a fórmula inmunomoduladora 5 a 7 días antes del procedimiento; $c$. los pacientes de alto riesgo nutricional, recibieron soporte nutricional (enteral o parenteral) y se informó al cirujano tratante para modificar la fecha de la intervención, según necesidad (Figura 1).

El éxito de la implementación de las intervenciones ERAS se midió con los indicadores de tiempo de estancia hospitalaria y porcentaje de complicaciones graves, siendo considerada en especial la presencia de fuga anastomótica. Estos datos fueron obtenidos de EIAS mensualmente y analizados en el comité del grupo ERAS y del grupo de consulta ERAS.

\section{Análisis estadístico}

Las variables cualitativas o categóricas fueron descritas mediante frecuencias y porcentajes y las cuantitativas a través de promedios, mediana, desviación estándar y rangos. Para el análisis por subgrupos se emplearon las variables de clasificación del estado nutricional (normal, riesgo de desnutrición o desnutrido) y grupos de edad ( $<60$ años y $\geq 60$ años). La comparación de porcentajes se hizo con la prueba $\mathrm{Chi}^{2}$ de Pearson y la comparación de medianas con la prueba de KruskalWallis; en los casos en los que no fue posible hacer la comparación, se reportó como "no calculado" (NC). Se utilizó el software estadístico STATA 12.

\section{Aspectos éticos}

El presente trabajo tuvo la aprobación del comité de investigación de la Clínica Reina Sofía. Los pacientes que ingresan al programa ERAS firman un consenti- 
miento informado avalado por el grupo de investigación y el comité de ética de la institución, en el cual permiten la utilización de sus datos con fines de auditoría e investigación.

\section{RESULTADOS}

Entre marzo de 2016 y diciembre de 2018 fueron ingresados 182 pacientes para cirugía colorrectal, de los cuales 1 no asistió a la consulta prequirúrgica. De los 181 pacientes restantes (100\%), $42 \%$ eran hombres, la edad promedio fue 63,9 años $( \pm 14,8)$ y el $14,9 \%$ de los participantes tenían 80 o más años. En cuanto al estado nutricional, $13,8 \%$ y $8,8 \%$ presentaron riesgo de desnutrición o estaban desnutridos respectivamente.

Respecto al IMC, el promedio fue $25,2 \pm 4$, el 17,1\% de los pacientes estudiados se clasificó en bajo peso, 48,1\% normales y $34,8 \%$ en sobrepeso. Comparando el estado nutricional con el IMC, se encontró que los pacientes con estado nutricional normal tuvieron una mediana, para IMC, en rango de sobrepeso y los pacientes con riesgo y desnutrición tuvieron los dos una mediana de

Tabla 1. Descripción de la población según el estado nutricional

\begin{tabular}{|c|c|c|c|c|c|}
\hline & $\begin{array}{c}\text { Normal } \\
n=140(77,4 \%)\end{array}$ & $\begin{array}{c}\text { Riesgo } \\
n=25(13,8 \%)\end{array}$ & $\begin{array}{c}\text { Desnutrido } \\
n=16(8,8 \%)\end{array}$ & $\begin{array}{c}\text { Total } \\
n=181(100 \%)\end{array}$ & Valor $p$ \\
\hline Sexo: Hombres ₹ & $62(44,3)$ & $7(28,0)$ & $7(43,8)$ & $76(42,0)$ & 0,31 \\
\hline Edad, en años $\dagger$ & $62.3(14,5)$ & $67,6(14,4)$ & $70,4(16,0)$ & $63,9(14,8)$ & 0,06 \\
\hline \multicolumn{6}{|l|}{ Grupo edad: ¥ } \\
\hline$<60$ años & $54(38,6)$ & $9(36,0)$ & $5(31,3)$ & $68(37,6)$ & 0,01 \\
\hline 60 a 79 años & $70(50,0)$ & $12(48,0)$ & $4(25,0)$ & $86(47,5)$ & \\
\hline$\geq 80$ años & $16(11,4)$ & $4(16,0)$ & $7(43,8)$ & $27(14,9)$ & \\
\hline \multicolumn{6}{|l|}{ Diagnósticos: ‡ } \\
\hline Diabetes & $13(9,3)$ & $3(12,0)$ & $2(12,5)$ & $18(9,9)$ & 0,86 \\
\hline Enfermedad cardiaca & $4(2,9)$ & $1(4,0)$ & $5(31,3)$ & $10(5,5)$ & $<0,01$ \\
\hline Enfermedad pulmonar & $10(7,1)$ & $1(4,0)$ & $3(18,8)$ & $14(7,7)$ & 0,19 \\
\hline \multicolumn{6}{|l|}{ Tratamientos previos: $\ddagger$} \\
\hline Inmunodepresor & $4(2,9)$ & $4(16,0)$ & $3(18,8)$ & $11(6,1)$ & $<0,01$ \\
\hline Quimioterapia & $6(4,3)$ & $4(16,0)$ & $2(12,5)$ & $12(6,6)$ & 0,05 \\
\hline Radioterapia & 0 & $1(4,0)$ & 0 & $1(0,6)$ & $\mathrm{NC}$ \\
\hline Peso preoperatorio $(\mathrm{Kg}) \dagger$ & $68,8(12,8)$ & $57,6(11,4)$ & $57,6(11,1)$ & $66.3(13.3)$ & $<0,01$ \\
\hline Cambio de peso preoperatorio $(\mathrm{Kg}) \dagger$ & $0,03(3,8)$ & $-5,9(2,7)$ & $-9,4(5,9)$ & $-1,6(5,0)$ & $<0,01$ \\
\hline $\mathrm{IMC}\left(\mathrm{Kg} / \mathrm{m}^{2}\right) \dagger$ & $26,0(3,7)$ & $22,4(3,6)$ & $21,7(3,3)$ & $25,2(4,0)$ & $<0,01$ \\
\hline \multicolumn{6}{|l|}{ Procedimiento quirúrgico realizado: ‡ } \\
\hline Cirugía abierta & $9(6,4)$ & $5(20,0)$ & $6(37,5)$ & $20(11,0)$ & $\mathrm{NC}$ \\
\hline Laparoscopia estándar & $116(82,9)$ & $16(64,0)$ & $8(50,0)$ & $140(77,3)$ & $<0,01$ \\
\hline Conversión de laparoscopia a abierta & $4(2,9)$ & $1(4,0)$ & 0 & $5(2,8)$ & $\mathrm{NC}$ \\
\hline Abordaje a través de estoma existente & $11(7,9)$ & $3(12,0)$ & $2(12,5)$ & $16(8,8)$ & $\mathrm{NC}$ \\
\hline
\end{tabular}

t: Valores presentados como mediana (percentil 25 y 75 )

‡:Valores presentados como frecuencia (\%)

NC: no calculado 
IMC en rango de normalidad (Tabla 1). La Figura 1 muestra los pacientes según el estado nutricional (bien nutridos, riesgo nutricional y desnutridos) y el estado ponderal según el IMC: bajo peso, normal o sobrepeso. Se encontró que $12 \%$ de los pacientes con riesgo de desnutrición tienen un IMC de sobrepeso y que más de la mitad (56,3\%) de los pacientes en estado de desnutrición tienen un IMC normal o en sobrepeso (Figura 2).

Las intervenciones prequirúrgicas predominantes fueron el uso de fórmulas inmunomoduladoras (76,8\%) y la suplementación por vía oral (13,8 \%), los pacientes desnutridos que requirieron repleción nutricional preoperatoria y no toleraron la vía oral o enteral recibieron nutrición parenteral (Tabla 2). En el mismo sentido, el tratamiento nutricional prequirúrgico predominante según la edad del paciente fue la inmunonutrición y los pacientes menores de 60 años fueron el grupo de edad que requirió mayor repleción nutricional por vía parenteral (Tabla 3).

En el periodo postoperatorio, 6,6 \% de los pacientes estudiados presentó íleo paralítico y 6,1 \% fuga anastomótica.

\section{DISCUSIÓN}

En cirugía colorrectal, el protocolo ERAS está actualmente bien establecido como la mejor forma de atención ${ }^{(15)}$. Las intervenciones nutricionales perioperatorias en el marco de este protocolo se centran en evitar el ayuno preoperatorio prolongado mediante la

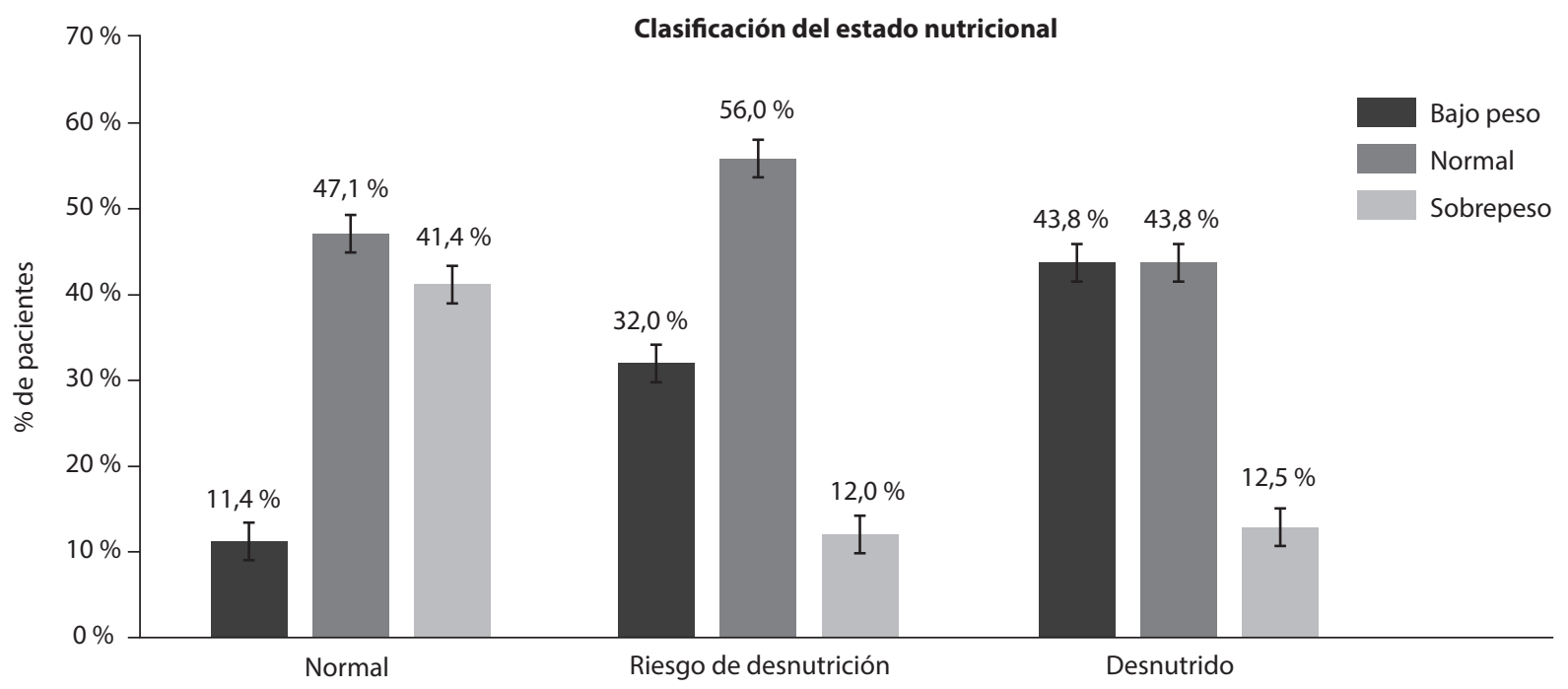

Figura 2. Clasificación del IMC según el estado nutricional.

Tabla 2. Intervenciones prequirúrgicas según el estado nutricional

\begin{tabular}{|c|c|c|c|c|}
\hline & $\begin{array}{c}\text { Normal } \\
n=140(77,4 \%)\end{array}$ & $\begin{array}{c}\text { Riesgo } \\
\mathrm{n}=\mathbf{2 5}(13,8 \%)\end{array}$ & $\begin{array}{c}\text { Desnutrido } \\
n=16(8,8 \%)\end{array}$ & $\begin{array}{c}\text { Total } \\
n=181(100 \%)\end{array}$ \\
\hline \multicolumn{5}{|l|}{ Tratamiento nutricional: ‡ } \\
\hline Alimentación habitual & $12(8,6)$ & 0 & 0 & $12(6,6)$ \\
\hline Suplemento VO & $16(11,4)$ & $7(28,0)$ & $2(12,5)$ & $14(13,8)$ \\
\hline Inmunonutrición VO & $112(80,0)$ & $18(72,0)$ & $9(56,3)$ & $139(76,8)$ \\
\hline Nutrición VP & 0 & 0 & $5(31,3)$ & $5(2,8)$ \\
\hline
\end{tabular}

‡:Valores presentados como frecuencia (\%)

VO: Vía oral; VP: vía parenteral 
carga oral de carbohidratos hasta dos horas antes de la cirugía y una intervención nutricional adaptada al riesgo nutricional. Estas intervenciones nutricionales perioperatorias son seguras y efectivas para reducir las complicaciones y la estadía postoperatoria. En este estudio se describen las intervenciones nutricionales prequirúrgicas realizadas a los pacientes adultos atendidos en la clínica Reina Sofía para cirugía colorrectal programada manejados dentro del programa ERAS.

La malnutrición prequirúrgica se ha considerado un factor de riesgo independiente para malos desenlaces en cirugía mayor ${ }^{(16)}$. Sin embargo, aún sigue siendo elevado el número de pacientes que llega a una intervención quirúrgica mayor con algún grado de desnutrición y sin intervención previa ${ }^{(17)}$. Es de resaltar que la intervención nutricional preoperatoria permite mejorar la condición del paciente al momento de la cirugía haciendo que las intervenciones sean factibles y costoefectivas $^{(18)}$. El presente estudio mostró que en la consulta prequirúrgica de pacientes de cirugía colorrectal se realizó la detección del riesgo nutricional en el marco del protocolo ERAS, encontrando una prevalencia de riesgo nutricional y desnutrición de $22,6 \%$, lo que se asemeja con lo encontrado en la literatura ${ }^{(19,20)}$.

Tabla 3. Intervenciones prequirúrgicas por grupos de edad

\begin{tabular}{|c|c|c|c|c|}
\hline & $\begin{array}{c}<60 \text { años } \\
n=68(37,6 \%)\end{array}$ & $\begin{array}{c}60 \text { a } 79 \text { años } \\
n=86(47,5 \%)\end{array}$ & $\begin{array}{c}\geq 80 \text { años } \\
\mathrm{n}=27(14,9 \%)\end{array}$ & Valor $p$ \\
\hline \multicolumn{5}{|l|}{ Diagnósticos: ‡ } \\
\hline Diabetes & 0 & $13(15,1)$ & $5(18,5)$ & $<0,01$ \\
\hline Enfermedad cardíaca & $3(4,4)$ & $2(2,3)$ & $5(18,5)$ & $<0,01$ \\
\hline Enfermedad pulmonar & $1(1,5)$ & $9(10,5)$ & $4(14,8)$ & 0,03 \\
\hline \multicolumn{5}{|l|}{ Tratamientos previos: $\neq$} \\
\hline Inmunodepresor & $9(13,2)$ & $2(2,3)$ & 0 & NC \\
\hline Quimioterapia & $8(11,8)$ & $4(4,7)$ & 0 & \\
\hline Radioterapia & 0 & $1(1,2)$ & 0 & \\
\hline Peso preoperatorio $(\mathrm{Kg}) \dagger$ & $67,2(13,1)$ & $66,8(13,0)$ & $62,5(14,4)$ & 0,18 \\
\hline Cambio de peso preoperatorio $(\mathrm{Kg}) \dagger$ & $-1,0(5,8)$ & $-1,6(4,3)$ & $-3,2(4,7)$ & 0,05 \\
\hline $\mathrm{IMC}\left(\mathrm{Kg} / \mathrm{m}^{2}\right) \dagger$ & $24,8(4,2)$ & $25,7(3,8)$ & $24,3(4,1)$ & 0,31 \\
\hline \multicolumn{5}{|l|}{ Tratamiento nutricional prequirúrgico: ‡ } \\
\hline Alimentación habitual & $5(7,4)$ & $5(5,8)$ & $2(7,4)$ & NC \\
\hline Suplemento VO & $4(5,9)$ & $16(18,6)$ & $5(18,5)$ & 0,05 \\
\hline Inmunonutrición VO & $56(82,4)$ & $64(74,4)$ & $19(70,4)$ & 0,35 \\
\hline Nutrición parenteral & $3(4,4)$ & $1(1,2)$ & $1(3,7)$ & NC \\
\hline \multicolumn{5}{|l|}{ Procedimiento quirúrgico realizado: ‡ } \\
\hline Cirugía abierta & $7(10,3)$ & $9(10,5)$ & $4(14,8)$ & 0,79 \\
\hline Laparoscopia estándar & $51(75,0)$ & $67(77,9)$ & $22(81,5)$ & 0,78 \\
\hline Conversión de laparoscopia a abierta & $1(1,5)$ & $4(4,7)$ & 0 & $\mathrm{NC}$ \\
\hline Abordaje a través de estoma existente & $9(13,2)$ & $6(7,0)$ & $1(3,7)$ & 0,23 \\
\hline
\end{tabular}

‡: valores presentados como frecuencia (\%)

†: valores presentados como promedio (+/- desviación estándar)

VO: vía oral; NC: no calculado 
La detección del riesgo nutricional por medio de herramientas de tamizaje aplicada por un profesional de la salud es el punto inicial del proceso de cuidado nutricional. En nuestra institución el nutricionista es quien la realiza el tamizaje nutricional; sin embargo, los resultados pueden variar respecto a instituciones donde sea realizada por el cirujano. Por ejemplo, Benoit et al. ${ }^{(21)}$ comparan los resultados de la identificación del riesgo nutricional en pacientes quirúrgicos realizada por cirujanos con la realizada por profesionales en nutrición, encontrando que los cirujanos subestimaron el puntaje en $29 \%$ de los pacientes y lo sobreestimaron en $21 \%$. Por lo anterior, la mejor estrategia siempre será el trabajo en colaboración entre los diferentes profesionales que participan en la atención del paciente y cada uno debe familiarizarse con las herramientas de tamización nutricional, aplicarlas en la consulta prequirúrgica y mejorar la detección del riesgo nutricional, lo que se capitaliza en una intervención nutricional oportuna y pertinente.

El uso de herramientas de tamizaje nutricional validadas como lo son el NRS 2002 y MST, ${ }^{(22,23)}$ puede ser útil en la identificación oportuna del riesgo nutricional durante la primera consulta prequirúrgica o en las primeras horas de hospitalización, llevando a la instauración de una terapia nutricional oportuna, como se evidencia en los resultados de esta investigación, que describe que todos los pacientes recibieron alguna intervención nutricional prequirúrgica.

Aunque el IMC es un factor de riesgo de mortalidad en pacientes con patología colorrectal ${ }^{(24)}$ no es una herramienta adaptada para identificar el riesgo nutricional ${ }^{(25)}$. En este estudio mostramos como el IMC no es útil para evaluar el riesgo de desnutrición prequirúrgica y puede subestimar la malnutrición al ser comparada con la evaluación del riesgo nutricional a través de herramientas validadas ${ }^{(26)}$. El IMC describe la distribución "normal" de la corpulencia de la población y sus extremos "anormales". Es por tanto de utilidad en la descripción del estado de corpulencia de la población. Sin embargo, no refleja el grado de adiposidad o de cantidad de masa muscular, es decir, no describe cambios en la composición corporal individualmente. Esta medida sigue siendo utilizada debido a que no existe otra forma tan rápida, fácil y poco costosa. En consecuencia, si un paciente tiene un IMC $>25 \mathrm{~kg} /$ $\mathrm{m}^{2}$ no significa que no esté en riesgo nutricional, y por lo tanto, es importante preguntar acerca de pérdida de peso reciente y hacer el cálculo del porcentaje de pérdida de peso. Es recomendable hacerlo en la primera consulta de los pacientes que serán sometidos a cirugía gastrointestinal mayor.

Según el protocolo ERAS, un paciente con cirugía programada que se encuentra en riesgo nutricional, debe tener una intervención que mejore su aporte calórico y proteico por lo menos 7 a 10 días previos a la cirugía $^{(27)}$. La mejor estrategia es el uso de suplementos nutricionales orales. En la práctica de la Reina Sofía, en el marco del protocolo ERAS, todos los pacientes en riesgo o en estado de desnutrición recibieron fórmulas inmunomoduladoras, suplementación por vía oral o nutrición parenteral. Como en el estudio de Kabata et al. el uso de suplementos nutricionales orales antes de cirugía en pacientes que no presentan malnutrición o han presentado pérdida de peso no considerada severa, mejora la condición física, ayuda a mantener un estado nutricional apropiado y reduce el número y la severidad de las complicaciones postoperatorias ${ }^{(28)}$. Si el paciente presenta un riesgo nutricional elevado y se detecta su incapacidad para cumplir los requerimientos por vía oral, debe considerarse la intervención con nutrición enteral o parenteral por un periodo de 10 a 14 días, según sus condiciones clínicas ${ }^{(12)}$. El tipo de soporte nutricional dependerá de la ruta por la cual el paciente pueda cubrir sus requerimientos calóricos y proteicos diarios en el preoperatorio ${ }^{(29)}$.

El uso de fórmulas inmunomoduladoras representa una situación particular, ya que no se limita al efecto puramente nutricional, porque con la adición de algunos nutrientes (arginina, ácidos grasos omega-3) se ejerce un efecto de modulación metabólico e inmunológico mejorando los desenlaces en el paciente quirúrgico $^{(2)}$. Por esto, son utilizadas en pacientes con o sin riesgo nutricional como parte de las estrategias de manejo perioperatorio. Son múltiples los metaanálisis publicados sobre este tema, en su mayoría a favor de las fórmulas inmunomoduladoras ${ }^{(12)}$. En el estudio de Moya y colaboradores ${ }^{(30)}$ sobre el uso de fórmulas inmonomoduladoras en pacientes de cirugía colorrectal bajo el protocolo ERAS, se encontró una disminución de la tasa de complicaciones, principalmente infecciosas, en los pacientes manejados con estrategias del protocolo ERAS y fórmulas inmunomoduladoras en el periodo perioperatorio.

\section{Limitaciones del estudio}

Esta investigación presenta algunas limitaciones que deben ser consideradas al momento de la interpretación o uso de los resultados. Primero, debido al tamaño de 
muestra y la baja incidencia de los eventos posquirúrgicos, no fue viable realizar análisis de asociación que permitieran determinar el efecto de las intervenciones prequirúrgicas sobre desenlaces clínicos relevantes, por ejemplo, la fuga anastomótica. Por tanto, se sugiere que próximas investigaciones sean de tipo multicéntrico, lo que permitirá aumentar el tamaño de muestra y disminuirá el tiempo de observación.

Segundo, debido a la ausencia de una cohorte histórica de pacientes previo a la instauración del protocolo ERAS, no es posible afirmar en cuanto mejoró la atención nutricional en la Clínica Reina Sofía. Sin embargo, se constata que desde la implementación del protocolo ERAS, todos los pacientes con cirugía colorrectal programada reciben intervención preoperatoria.

\section{CONCLUSIÓN}

La implementación del protocolo ERAS en la Clínica Reina Sofía permitió que todos los pacientes adultos sometidos a cirugía colorrectal tuvieran una intervención nutricional prequirúrgica. La intervención más frecuente fue el uso de suplementos nutricionales por vía oral, en especial fórmulas inmunomoduladoras. La nutrición por vía parenteral se requirió exclusivamente en pacientes con desnutrición. Se sugieren estudios que permitan valorar el impacto de las intervenciones de este protocolo sobre los desenlaces de los pacientes.

\section{Agradecimientos}

Agradecemos al grupo ERAS Clínica Reina Sofía por el apoyo en la publicación y el análisis de los datos.

\section{Financiación}

La investigación no tuvo financiación.

\section{Conflicto de intereses}

Los autores declaran no tener conflicto de intereses.

\section{Declaración de autoría}

AN participó en la redacción del artículo, la concepción, realización y desarrollo de la investigación, análisis e interpretación de los resultados. IP participó en la concepción del artículo, el desarrollo de la investigación, la obtención de datos y el análisis de resultados. GD participó en el análisis de los datos, interpretación de la información y redacción del artículo. Todos los autores revisaron y aprobaron la versión final del manuscrito.

\section{Referencias bibliográficas}

1. Butterworth CE. The skeleton in the hospital closet. Nutrition Today. 1974;9:4-8.

2. Klek S, Szybinski P, Szczepanek K. Perioperative immunonutrition in surgical cancer patients: A summary of a decade of research. World J Surg. 2014;38(4):803-12.

3. Waitzberg DL, Saito H, Plank LD, Jamieson GG, Jagannath P, Hwang TL, et al. Postsurgical infections are reduced with specialized nutrition support. World J Surg. 2006;30(8):1592-604.

4. Tappenden KA, Quatrara B, Parkhurst ML, Malone AM, Fanjiang G, Ziegler TR. Critical Role of Nutrition in Improving Quality of Care. JPEN J Parenter Enter Nutr. 2013;37(4):482-97.

5. Slinde F, Grönberg AM, Svantesson U, Hulthén L, Larsson S. Energy expenditure in chronic obstructive pulmonary disease-evaluation of simple measures. Eur J Clin Nutr. 2011;65(12):1309-13.

6. Evans D, McNamara L, Maskew M, Selibas K, Van Amsterdam $\mathrm{D}$, Baines $\mathrm{N}$, et al. Impact of nutritional supplementation on immune response, body mass index and bioelectrical impedance in HIV-positive patients starting antiretroviral therapy. Nutrition Journal. 2013;12:111.

7. Kourkouta L, Monios A, Iliadis C, Ouzounakis P. AIDS and nutrition in patients. Prog Heal Sci. 2017;7(1):0-0.

8. Zamora T, Klaber I, Urrutia J. Hip fracture in the elderly. Clin Med Insights Geriatr. 2017;10:1-10.

9. D FBM, D LMM, Ph D. Perioperative nutritional support of patients undergoing pancreatic surgery in the age of ERAS. Nutrition. 2014;30(11-12):1267-71. doi.org/10.1016/j. nut.2014.03.002

10. Kassin MT, Owen RM, Perez SD, Leeds I, Cox JC, Schnier K, et al. Risk Factors for 30-Day Hospital Readmission among General Surgery Patients. J Am Coll Surg. 2012;215(3):32230. doi: 10.1016/j.jamcollsurg.2012.05.024.

11. Ljungqvist O, Dardai E, Allison SP. Basics in Clinical Nutrition: Perioperative nutrition. e-SPEN. 2010;5(2):e936. doi.org/10.1016/j.eclnm.2009.06.011

12. Weimann A, Braga M, Carli F, Higashiguchi T, Hübner M, Klek S, et al. ESPEN guideline: Clinical nutrition in surgery. Clin Nutr. 2017;36(3):623-50.

13. Perry R, Scott LJ, Richards A, Haase AM, Savovi J, Ness AR, et al. Pre-admission interventions to improve outcome after elective surgery - protocol for a systematic review. Syst Rev. 2016;23(5):88. doi: 10.1186/s13643-016-0266-9.

14. Organización Panamericana de la Salud, Organización Mundial de la Salud. Guía clínica para atención primaria a las personas adultas mayores. Promoción de salud y envejecimiento activo. Serie de materiales de capacitación. Número 1. 2002;10. Available from: http://www.sld.cu/galerias/pdf/ sitios/gericuba/introduccion.pdf

15. Pędziwiatr M, Mavrikis J, Witowski J, Adamos A, Major P, Nowakowski $M$, et al. Current status of enhanced recovery 
after surgery (ERAS) protocol in gastrointestinal surgery. Med Oncol. 2018;35(6):1-8. http://dx.doi.org/10.1007/s12032018-1153-0

16. Braga M, Ljungqvist $\mathrm{O}$, Soeters $\mathrm{P}$, Fearon $\mathrm{K}$, Weimann A, Bozzetti F. ESPEN Guidelines on Parenteral Nutrition: Surgery. Clin Nutr. 2009;28(4):378-86. http://dx.doi. org/10.1016/j.clnu.2009.04.002

17. Wischmeyer PE, Carli F, Evans DC, Guilbert S, Kozar R, Pryor A, et al. American Society for Enhanced Recovery and Perioperative Quality Initiative Joint Consensus Statement on Nutrition Screening and Therapy Within a Surgical Enhanced Recovery Pathway. Anesth Analg. 2018;126(6):1883-1895. doi: 10.1213/ANE.0000000000002743.

18. Scott M, Martindale R. Perioperative Nutrition: A High-Impact, Low-Risk, Low-Cost Intervention. Anesth Analg. 2018;126(6): 1803-1804. doi: 10.1213/ANE.0000000000002911.

19. Leandro-MerhiVA,AquinoJLB.Determinants of Malnutrition and Postoperative Complications in Hospitalized Surgical Patients. J Health Popul Nutr. 2014 Sep; 32(3): 400-410.

20. Shpata V, Prendushi X, Kreka M, Kola I, Kurti F, Ohri I. Malnutrition at the Time of Surgery Affects Negatively the Clinical Outcome of Critically Ill Patients with Gastrointestinal Cancer. Med Arch. 2014;68(4):263-7. doi: 10.5455/medarh.2014.68.263-267.

21. Benoit M, Grass F, Demartines N, Coti-Bertrand P, Schäfer M, Hübner M. Use of the nutritional risk score by surgeons and nutritionists. Clin Nutr. 2016;35(1):230-3.

22. Kondrup J, Allison SP, Elia M, Vellas B, Plauth M. ESPEN guidelines for nutrition screening 2002. Clin Nutr. 2003;22(4):415-21.

23. Ferguson M, Capra S, Bauer J, Banks M. Development of a valid and reliable malnutrition screening tool for adult acute hospital patients. Nutrition. 1999;15(6):458-64. dx.doi. org/10.1016/S0899-9007(99)00084-2

24. Shaukat A, Dostal A, Menk J, Church TR. BMI Is a Risk Factor for Colorectal Cancer Mortality. Dig Dis Sci. 2017;62(9):2511-7.

25. Basdevant A, Clément K, Oppert JM. Vers de nouveaux phénotypes et de nouvelles nosographies: De l'obésité aux maladies du tissu adipeux. Obesite. 2013;8(4):234-43.

26. Thieme RD, Cutchma G, Eliana M, Chieferdecker M, Carlos A, Campos L. Nutritional risk index is predictor of postoperative complications in operations of digestive system or abdominal wall? Arq Bras Cir Dig. 2013;26(4):286-92. doi: $10.1590 /$ s0102-67202013000400007

27. Gustafsson UO, Scott MJ, Hubner M, Nygren J, Demartines $\mathrm{N}$, Francis N, et al. Guidelines for Perioperative Care in Elective Colorectal Surgery: Enhanced Recovery After Surgery $\left(\right.$ ERAS $^{\circledast}$ ) Society Recommendations: 2018. World J Surg. 2019;43(3):659-95. doi: org/10.1007/s00268-018-4844-y

28. Kabata P, Jastrzębski T, Kąkol M, Król K, Bobowicz M, Kosowska A, et al. Preoperative nutritional support in cancer patients with no clinical signs of malnutrition-prospective randomized controlled trial. Support Care Cancer. 2015;23(2):365-70.

29. Martindale RG, McClave SA, Kozar RA, Heyland DK, Miller $\mathrm{KR}$, Wischmeyer PE, et al. An Evidence-Based Approach to Perioperative Nutrition Support in the Elective Surgery Patient. JPEN J Parenter Enter Nutr. 2013;37:39S-50S.

30. Moya P, Soriano-Irigaray L, Ramirez JM, Garcea A, Blasco $\mathrm{O}$, Blanco FJ, et al. Perioperative standard oral nutrition supplements versus immunonutrition in patients undergoing colorectal resection in an Enhanced Recovery (ERAS) protocol. Medicine. 2016;95(21):1-11. doi:10.1097/ MD.0000000000003704 